species between the Permian and the end of the Middle Triassic (between 251.9 million and around 237 million years ago) has focused on the effects of a global trend towards aridification. It was proposed that, after a worldwide collapse of plant communities and a mass extinction of species that cascaded through the food chain ${ }^{9}$, there was a change in the floral species across global landscapes by the Middle Triassic period. For the demise of Glossopteris, Fielding and colleagues find no evidence of an aridification trend in their region that would suggest that a hot terrestrial landscape promoted a mass extinction of plants during the time of the end-Permian crisis.

This conclusion of Fielding and colleagues' regional work is supported by a comprehensive analysis of plant fossil records on a global scale conducted by Nowak and colleagues. The authors analysed the patterns of previously reported plant fossils from $259.1 \mathrm{mil}$ lion to around 237 million years ago, which spans the end-Permian mass extinction and the Early and Middle Triassic. They generated a database that includes information on more than 7,300 plant macrofossils and nearly 43,000 fossil records of pollen or spores. So far, this is the most comprehensive database generated for floral analysis before and after the end-Permian crisis. It amasses the evidence that has been considered by many palaeontologists to indicate a trend in mass extinction of terrestrial plants that mirrors that of the marine mass extinction ${ }^{9}$.

The authors present origination, extinction and turnover patterns at the level of species and genera on a stage-by-stage basis (stages being steps in the geological timescale). The diversity of genera was relatively constant across the time interval, although the species diversity of macrofloral fossils dropped 251.9 million years ago. The diversity of genera represented by spores and pollen remained constant across the time frame studied, although Nowak et al. note a small decline in species-level diversity around 251.9 million years ago. Of the groups of plants that have either pollen or spores, the spore-bearing ferns, as well as the pollenproducing seed ferns and cycads, declined in diversity during this time, whereas the pollen-bearing conifers and ginkgos increased in diversity.

In contrast to prevailing wisdom, Nowak and colleagues demonstrate that land plants did not experience widespread extinction during Earth's most severe biological crisis. Their conclusion is similar to that drawn for terrestrial vertebrates ${ }^{11}$. This leaves the relationship between the end-Permian marine mass extinction and the effect on land at the time enigmatic for now, and still up in the air for further investigation.

Robert A. Gastaldo is in the Department of Geology, Colby College, Waterville, Maine 04901, USA.

e-mail:ragastal@colby.edu
1. Erwin, D. Sci. Am. 275, 72-78 (1996).

2. Burgess, S. D., Bowring, S. \& Shen, S. Z. Proc. Natl Acad. Sci. USA 111, 3316-3321 (2014)

3. Payne, J. L. \& Clapham, M. E. Annu. Rev. Earth Planet. Sci. 40, 89-111 (2012).

4. Fielding, C. R. et al. Nature Commun. 10, 385 (2019).

5. Nowak, H., Schneebeli-Hermann, E. \& Kustatscher, E. Nature Commun. 10, 384 (2019)

6. Behrensmeyer, A. K., Kidwell, S. M. \& Gastaldo, R. A. Paleobiology 26, 103-147 (2000).
7. Gastaldo, R. A., Neveling, J., Geissman, J. W. \& Kamo. S. L. Geol. Soc. Am. Bull. 130, 1411-1438 (2018).

8. Collinson, J. W., Hammer, W. R., Askin, R. A. \& Elliot, D. H. Geol. Soc. Am. Bull. 118, 747-763 (2006)

9. Benton, M. J. Phil. Trans. R. Soc. A 376, 20170076 (2018).

10.Smith, R. H. M. \& Botha-Brink, J. Palaeogeogr. Palaeoclimatol. Palaeoecol. 396, 99-118 (2014).

11.Sues, H. D. \& Fraser, N. C. Triassic Life on Land: The Great Transition (Columbia Univ. Press, 2010).

\title{
Materials in flatland twist and shine
}

\section{Four studies demonstrate the vast opportunities provided by stacking pairs of monolayer materials and changing the resulting optical properties by twisting one material with respect to the other. SEE LETTERS P.66, P.71, P.76 \& P.81}

\section{BERNHARD URBASZEK \& AJIT SRIVASTAVA}

A tomically thin materials are currently being investigated for fundamental research and applications in optics and electronics, because they interact strongly with light and have fascinating magnetic properties. When two different monolayer materials are brought into contact to form a bilayer, electrons can no longer move freely in the planes of the atomic layers. Instead, they are trapped in spatially periodic potentialenergy variations called moiré potentials, as a result of interactions between the layers ${ }^{1}$. These nanometre-scale potentials are caused by the layers having different orientations or lattice constants - parameters that describe the dimensions of a unit cell in a crystal lattice. Moiré potentials have been predicted to strongly modify the optical properties of such bilayers ${ }^{2}$. Four papers in this issue $e^{3-6}$ report observations of optical emission and absorption that confirm this prediction.

Monolayers of materials called transitionmetal dichalcogenides (TMDs) have strong in-plane covalent bonds, and can therefore be produced by exfoliation (the removal of sheets from a bulk crystal) in a similar way to graphene - a single layer of carbon atoms. However, unlike graphene, atomically thin TMD crystals are semiconductors that have an energy gap between their higher-energy (conduction) and lower-energy (valence) electronic bands. Consequently, charge carriers in these materials that are excited by light or that are injected using a voltage can relax from the conduction band to the valence band by emitting particles of light (photons).

Just as the weak attraction between layers lends itself to exfoliation, it also allows two monolayers of different TMDs (such as tungsten disulfide and molybdenum diselenide) to be stacked on top of each other to form what is known as an artificial heterobilayer (Fig. 1a). If the layers are periodic crystals that have slightly different lattice constants, the electronic properties of each layer are modified by the presence of the other layer. Specifically, the electronic states and band structure of the heterobilayer depend on the spacing and relative alignment of the atoms.

Because of the slight mismatch in the lattice constants of the two layers, arrangements of atoms in the heterobilayer change periodically: atoms of metals (such as molybdenum and tungsten) are positioned on top of each other at certain points, whereas atoms of chalcogens (such as sulfur and selenium) are aligned at other points. These different configurations of atoms, known as registries, result in different energies for the valence and conduction bands, as verified by scanning tunnelling microscopy ${ }^{1}$. Consequently, electrons in the plane of the heterobilayer are subject to periodically changing bands, and, if the variations in the band energies are sufficiently large, electrons are trapped in moiré potentials.

An attractive feature of such heterobilayers is the tunability of their periodic potential. Adding a slight twist during the stacking process, or exchanging one lattice with another that has a different lattice constant, leads to moiré potentials that have a different spatial periodicity. This feature enables the electronic properties of layered materials to be tailored in a fundamentally new way. As a result, heterobilayers are becoming a playground for exploring exotic quantum phenomena - rather like how the strong interactions between electrons in twisted bilayers of graphene have led to the spectacular observation of superconductivity in electronic-transport studies ${ }^{7}$.

The four current papers investigate the impact of moiré potentials on light emission 
a

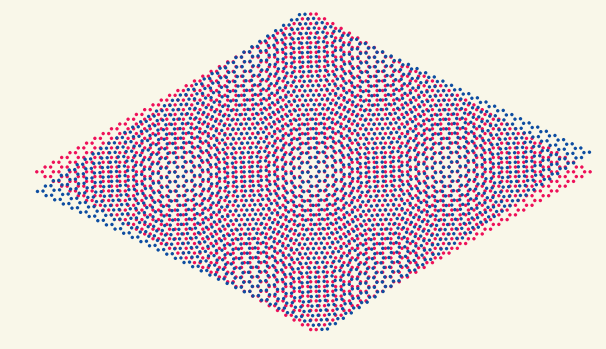

b

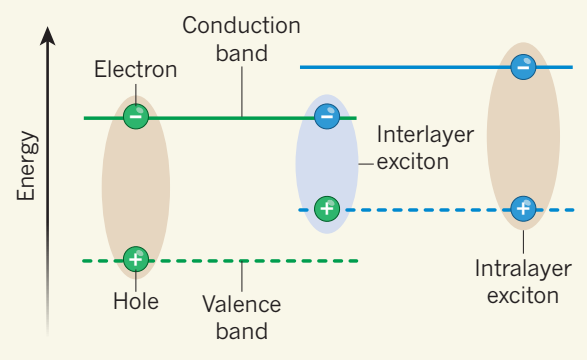

c

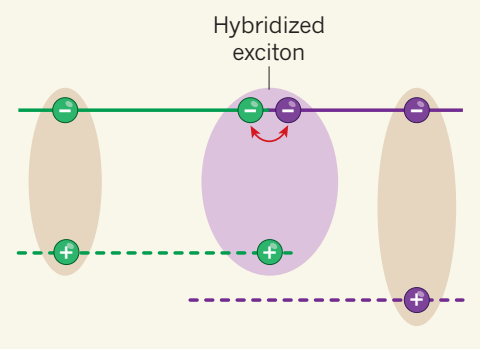

Figure 1 | Features of moiré heterobilayers. a, Four papers ${ }^{3-6}$ report on the optical properties of moiré heterobilayers - stacks of two different monolayer materials that display a 'moiré' pattern. b. The teams studied heterobilayers in which light emission and absorption are governed by electrons and holes (electron vacancies) forming bound states called excitons. Tran et al. ${ }^{3}$ and Seyler et al. ${ }^{4}$ studied molybdenum diselenidetungsten diselenide heterobilayers. Shown here are electronic-band diagrams for molybdenum diselenide (green) and tungsten diselenide (blue) that illustrate the higher-energy (conduction) and lower-energy (valence) bands.
The two groups explored interlayer excitons, in which the electron and hole exist in different layers of the heterobilayer (indicated by the different colours of the charge carriers). Jin et al. ${ }^{5}$ focused on intralayer excitons, in which the electron and hole reside in the same layer. c, Alexeev et al. ${ }^{6}$ found that intralayer excitons can be mixed (hybridized) with interlayer excitons in heterobilayers of molybdenum diselenide and tungsten disulfide, the band diagram of which is shown in purple. The conduction band is delocalized over both layers, which permits the existence of a hybridized electron state (indicated by the arrow). and absorption in heterobilayers of TMDs. The work has many new aspects compared with studies on graphene-boron nitride heterobilayers ${ }^{1}$. First, the variation in the band energies for the different atomic registries is larger. Second, light emission ${ }^{3,4}$ and absorption $^{5,6}$ in the TMD structures are governed by electrons and holes (electron vacancies) forming bound states called excitons ${ }^{8}$. And third, the excitons interact with light of a specific polarization that depends on the local symmetry of the atoms arranged periodically in the heterobilayer.

Tran et al. ${ }^{3}$ (page 71) explored interlayer excitons, in which the electron and hole reside in different layers (Fig. 1b). The authors obtained emission spectra from molybdenum diselenide-tungsten diselenide that had a small twist angle between the layers. They located peaks in the spectra that corresponded to interlayer excitons, and found that the energy separation between the peaks and the polarization of the emission depended on the twist angle. These results indicate that it might be possible to achieve nanoscale patterning of optical and magnetic properties in heterobilayers ${ }^{2,4}$.

Seyler et al. ${ }^{4}$ (page 66) studied individual interlayer excitons trapped in moiré potentials, also in molybdenum diselenide-tungsten diselenide structures. They detected lines in emission spectra that corresponded to highly polarized light and that split into multiple peaks when magnetic fields were applied. These emission lines provide a fingerprint of the magnetic moment and valley - minimum or maximum of the band structure - of the electron and hole that formed a particular exciton. However, to verify that the emission really stems from a single exciton trapped in a specific moiré potential, measurements based on a phenomenon called photon antibunching will be necessary, to prove that, as expected, only one photon is emitted at a time?
Whereas Tran et al. and Seyler et al. focused on light emission, Jin et al. ${ }^{5}$ (page 76) detected experimental signatures of changes in light absorption caused by moiré potentials in tungsten diselenide-tungsten disulfide heterobilayers. The authors examined intralayer excitons ${ }^{10}$, in which the electron and hole exist in the same layer (Fig. 1b). They showed that the strong, intriguing absorption features associated with these excitons can be tuned by an applied voltage.

Finally, Alexeev et al. ${ }^{6}$ (page 81 ) found evidence that intralayer excitons can be mixed (hybridized) with interlayer excitons in molybdenum diselenide-tungsten disulfide heterobilayers, because the conduction band is delocalized over both layers (Fig. 1c). They showed that this feature amplifies the impact of the moiré potentials on optical properties. These hybridized states could combine the strong optical absorption of intralayer excitons with the tunability of interlayer excitons in electric fields - this tunability is attributable to the permanent electric dipole of interlayer excitons, which results from the electron and hole being in different layers.

These studies and others ${ }^{10-12}$ give a first glimpse of the opportunities offered by combining two materials and changing the resulting optical properties simply through the twist angle. A challenge will be to control the natural tendency of two stacked monolayers to rotate after deposition towards an energetically favourable configuration, as has been observed for graphene-boron nitride heterobilayers ${ }^{13}$. This local rearrangement of atoms, termed reconstruction, can result in deviations from a completely periodic potential and therefore inhomogeneities in the moiré potentials ${ }^{14}$. Further insight into this issue could be gained by studying the differences that might occur between exfoliated heterobilayers and samples grown by a technique called chemical-vapour deposition $^{15}$.
In these four papers, an optical microscope with a spatial resolution of typically 1 micrometre was used to investigate moiré potentials that, by contrast, have a periodicity of only tens of nanometres. By using 'near-field' techniques that have a spatial resolution of the order of 10 nanometres, individual potentials could be addressed ${ }^{16}$. Depending on the size and depth of the moiré potentials, the number of trapped excitons could really go down to one, offering a source of single photons. Finally, the potentials themselves could provide a periodic array of tunable light emitters - one of the many fascinating prospects for optics in twodimensional 'flatland', although, admittedly, now the material is not completely flat, but two monolayers thick.

Bernhard Urbaszek is at the Laboratory of Physics and Chemistry of Nano-Objects, INSA-CNRS-UPS, Toulouse University, Toulouse 31077, France. Ajit Srivastava is in the Department of Physics, Emory University, Atlanta, Georgia 30322, USA.

e-mails: urbaszek@insa-toulouse.fr; ajit.srivastava@emory.edu

1. Zhang, C. et al. Sci. Adv. 3, e1601459 (2017).

2. Yu, H., Liu, G.-B., Tang, J., Xu, X. \& Yao, W. Sci. Adv. 3, e1701696 (2017).

3. Tran, K. et al. Nature 567, 71-75 (2019).

4. Seyler, K. L. et al. Nature 567, 66-70 (2019).

5. Jin, C. et al. Nature 567, 76-80 (2019).

6. Alexeev, E. M. et al. Nature 567, 81-86 (2019).

7. Cao, Y. et al. Nature 556, 43-50 (2018)

8. Wang, G. et al. Rev. Mod. Phys. 90, 021001 (2018).

9. Michler, P. et al. Science 290, 2282-2285 (2000).

10.Zhang, N. et al. Nano Lett. 18, 7651-7657 (2018).

11.Ciarrocchi, A. et al. Nature Photon. 13, 131-136 (2019).

12.Paik, E. Y. et al. Preprint at https://arxiv.org/ abs/1901.00598 (2019).

13.Woods, C. R. et al. Nature Commun. 7, 10800 (2016).

14.Alden, J. S. et al. Proc. Natl Acad. Sci. USA 110, 11256-11260 (2013).

15. Hsu, W.-T. et al. Nature Commun. 9, 1356 (2018).

16.Schmidt, P. et al. Nature Nanotechnol. 13 1035-1041 (2018). 